\title{
Conclusions
}

The 23 Case Studies in the LEARN Toolkit span, but expand on, all seven themes of the original LERU Roadmap. ${ }^{1}$ Overall, they underline the challenges and opportunities identified in the 2013 document, but now offer solutions to address a range of issues. They are grouped as follows:

- Policy and Leadership

- Advocacy

- Subject approaches

- Open Data

- Research Data Infrastructure

- Costs

- Roles, Responsibilities and Skills

- Tool development

\section{POLICY AND LEADERSHIP}

The LERU Roadmap advocated that 'Every LERU member should develop and promulgate an institutional data policy'. ${ }^{2}$ The LEARN Toolkit provides the tools to do this, with a model RDM policy and guidance in Part 2 of the compilation. Additionally, the Case Studies support the call for policy leadership and alignment. Case Study 1 from the Wellcome Trust argues that there is broad agreement on policy amongst research funders on the importance of RDM, whilst identifying key challenges which remain. The Executive Briefings in six languages in Part 3 are designed for senior decision makers, to support them in delivering sound solutions. Case Study 2 describes the process of developing a model RDM policy for Austria, based on the LEARN template, which acts as a framework and which can be customised at a local level. Case Study 3 looks at Brexit and its potential impact on Open Science, concluding that perhaps the greatest threat currently lies in a possible lack of engagement in the UK with the European Open Science Cloud. Case Study 4 looks at linking the practice of RDM with research integrity frameworks.

\section{ADVOCACY}

Many of the Case Studies are devoted to the theme of advocacy. The LERU Roadmap stressed that LERU members, researchers and research funders should 'Promote best practice in data management, citation and interoperability to increase the visibility of data'. ${ }^{3}$ This is true of the Case Studies from both Latin America/the Caribbean and Europe. Some interesting themes around advocacy are identified. Case Study 5 makes the point that RDM advocacy to researchers is in its infancy. Accordingly, qualitative rather than quantitative measures and approaches currently predominate. However, the institution in this Case Study has undertaken a wide-ranging internal survey which will provide a baseline for future activity. Case Study 6 emphasises that what is needed is to identify RDM stakeholders, ensure good communication, and develop implementation plans. Case Study 7 links leadership and advocacy by asking the question 'Who has leadership for RDM at an institutional level in the University of the West Indies?'. Case Study 8 underlines the challenges involved in RDM advocacy. In this institution, after years of activity, the difficulties in changing institutional culture with regard to RDM remain. 
The Case Studies in the LEARN Toolkit look particularly at RDM issues in the Arts, Humanities and Social Sciences. Case Study 9 looks in detail at the challenges and opportunities at UCL (University College London). It identifies that many researchers in these disciplines do not use, or are unaware of, UCLsupported RDM solutions and that there is a need for advocacy to these communities. Case Study 10 is from the Performing Arts. Discussion on RDM has centred on the sciences (in the English sense, excluding the Arts, Humanities and Social Sciences). Because of how Arts projects are funded and structured, there are special problems and challenges relating to RDM - which this Case Study identifies.

\section{OPEN DATA}

The issue of Open Data is treated in several Case Studies. Case Study 11 argues that research is an inherently Open enterprise and that universities increasingly see Open Data as part of their future. Case Study 12 looks at Open Data in educational resources, showing that there is a place for research data in taught provision, where the university supports research-based or research-led education. Case Study 13 , on the other hand, shows how closed data is managed in a practical way in the University of the Andes in Colombia.

\section{RESEARCH DATA INFRASTRUCTURE}

A number of the Case Studies look at the provision of infrastructure. Case Study 14 from UCL (University College London) draws lessons from its Research Data Storage service. Case Study 15 analyses a collaborative RDM service in Brazil, using the Dataverse network. Case Study 16 looks at the recent Report from the European Commission's High Level Expert Group on the European Open Science Cloud and discusses the vision for that development, based on research data which is FAIR (Findable, Accessible, Interoperable and Re-usable).

costs

LEARN itself is not an economic study, but several of the Case Studies look at RDM costs. Case Study 14 for example, gives some detailed costings for the Research Data Storage service at UCL (University Colleg London). Case Study 16 looks at the projected costs for delivering the European Open Science Cloud. Case Study 17 from the University of Edinburgh gives detailed costings and analysis for the formation of its research data services.

\section{ROLES, RESPONSIBILITIES AND SKILLS}

A number of the Case Studies emphasise the need for training and skills development for all stakeholders in the RDM landscape. Case Study 18 looks at training for early career researchers by analysing the 2016 LERU Doctoral Summer School. Case Study 19 looks at training subject liaison librarians in research data management. Chapter 20 looks at the EDISON project, which is creating a data science profession for Europe.
A number of chapters look at tool development to support RDM. Case Study 21 looks at legal requirements and shows how the use of licences can establish frameworks for sharing, re-use and compliance. Case Study 22 looks to Argentina and the development of Data Management planning, concluding that good Data Management Plans will deliver good research. Finally, chapter 23 looks at the LEARN Readiness survey. The survey allows research performing institutions to assess their level of preparation for RDM by answering 13 questions. Using a traffic light marking scheme of red, amber or green, the survey will be marked and enable those taking the test to see how prepared they are. The test can be taken iteratively, so that over a period an institution can measure its progress in RDM activity.

\section{CONCLUSION}

Research data is the new currency of the digital age. From sonnets to statistics, and genes to geodata, the amount of material being created and stored is growing exponentially. The LERU Roadmap identifies serious gap in the level of preparation amongst research performing organisations. This gulf is prominent in areas such as policy development, awareness of current issues, skills development, training, costs, community building, governance, disciplinary/legal/terminological and geographical differences. The LEARN Toolkit is designed to identify sound solutions and proposals for these challenges and opportunities. By adopting recommended LEARN practices, templates and guidance, all those involved as stakeholders in RDM can introduce best practice into their institutions. 